Bangladesh J. Bot. 50(3): 887-894, 2021 (September) SpecialＤOI: https://doi.org/10.3329/bjb.v50i5.56441

\title{
TRANSCRIPTOME ANALYSIS OF THE IMMATURE INFLORESCENCES OF FOXTAIL MILLET TREATED WITH SQ-1 FOR INDUCING MALE STERILITY
}

\author{
Chunfang Wang ${ }^{1}$, Dongmei Qi ${ }^{1}$, Wei Zhang ${ }^{2}$, Yongeiang Wang ${ }^{3}$ \\ ShenkUI ShI ${ }^{1}$ AND XIANMIN DiaO ${ }^{2 *}$ \\ College of Biological and Food Science, Hebei Normal University for Nationalities, \\ Chengde, China,
}

Keywords: Foxtail millet, Male sterility, Chemical hybridizing agent (CHA), Transcriptome, SQ-1

\begin{abstract}
A comparative transcriptomic analysis was carried out through next-generation sequencing technology for the SQ-1-treated immature inflorescences of foxtail millet (Setaria italica). It was found that $96.7 \%$ of the pollen of SQ-1-treated plants were sterile, thereby confirming the efficacy of SQ-1 in inducing male sterility in foxtail millet. A total of 6659 DEGs were identified from the plants of 2 nd days of treatment, among which 3532 and 3127 were up- and down-regulated, respectively. Comparatively fewer DEGs, 1584 and 2654, were detected from the plants of 4th and 6th days of treatment, respectively. The gene expression profiles of foxtail millet were verified through qRT-PCR in accordance to 16 previously reported genes from other crops that were associated with pollen development. Reference genes Si002651 and Si002929 were found to be the most proper option for SQ-1 treatment.
\end{abstract}

\section{Introduction}

Setaria italica (L.) P. Beauv. belonging to Poaceae commonly known as Foxtail millet, is an important cereal grain and forage crop, which is predominantly cultivated in North China. It is a self-pollinated crop with a tiny floral morphology and delicate anthesis behavior, which represent a major bottleneck in artificial hybridization for breeding progress and the utilization of heterosis. Besides, the lack of an efficient method for artificial hybridization has also hindered the development of this plant as a model system. The production of hybrid seed is primarily dependent on the breeding performance of male sterile lines in the application of heterosis in foxtail millet (Wang et al. 2002). Till now, no cytoplasmic male sterile or photoperiod- and thermo-sensitive genic male sterile lines are available for foxtail millet. Because of these restrictions, chemical hybridizing agents (CHAs) appear to be an ideal and effective alternative. SQ-1 has been demonstrated to be an ideal chemical male gametocide for foxtail millet, owing to its high efficacy, among the chemical male gametocides to generate male sterile lines (Wang et al. 2003, Zhu et al. 2015, Wang et al. 2016). Furthermore, Song et al. (2011) reported that a suitable dosage of SQ-1 sprayed at an appropriate time can result in male sterility between 95 and 100\% and thereby it can be applied for artificial hybridization in foxtail millet.

In the present study, a comparative transcriptomic analysis was carried out for the SQ-1treated immature inflorescences of foxtail millet with the objective of elucidating the molecular mechanisms underlying the induction of male sterility. Accordingly, 6659, 1584, and 2654 genes were identified that were differentially expressed (DEGs) on 2nd, 4th and 6th days after SQ-1

*Author for correspondence: <diaoxianmin@caas.cn>;<shishenkui@126.com>. ${ }^{1}$ College of Biological and Food Science, Hebei Normal University for Nationalities, Chengde, China ${ }^{2}$ Institute of Crop Sciences, Chinese Academy of Agricultural Science, Beijing, China. ${ }^{3}$ Institute of Cotton, Hebei Academy of Agriculture and Forestry Sciences, Shijiazhuang, China. 
treatment, respectively. Later on, these genes were classified into different functional pathways based on GO analysis. Thus, based on the experimental data, the present investigation aimed to examine the molecular mechanisms related to anther and pollen development for molecular breeding in foxtail millet.

\section{Materials and Methods}

In the present study, Yugu 1 cultivar of foxtail millet was selected for spraying with a $4 \%$ solution of SQ-1 ( Song et al. 2011, Wang et al. 2019) at the eight-leaf stage. The plants were grown in experimental field in Chengde city, Hebei province, China under natural conditions. The solution of SQ-1 was sprayed onto plants, after SQ-1 treatment, the plants were properly labeled and the panicles were bagged prior to flowering. At the time of flowering, pollen grains were examined to determine the extent of male sterility, and grain number was subsequently used to assess the actual seed setting rate (Song et al. 2011). Total RNA was extracted from composite samples from three biological replicates using Trizol (Invitrogen). The quality of the extracted RNA was determined using a NanoDrop spectrophotometer and Agilent 2100 Bioanalyzer (Agilent, Santa Clara, CA, USA), mRNA was isolated following the protocol of the NEBNext ${ }^{\circledR}$ Poly (A) mRNA Magnetic Isolation Module (NEB, Ipswich, MA, USA).

Sequencing libraries were created using an NEBNext ${ }^{\circledR}$ Ultra $^{\mathrm{TM}}$ RNA Library Prep Kit for Illumina. High-quality libraries were sequenced using an Illumina HiSeq X sequencer (Illumina, San Diego, CA, USA) and the data output of each sample was found to be greater than $6 \mathrm{~Gb}$. To remove the low-quality reads, the adapter sequences were filtered out, and used SolexaQA (http://solexaqa.sourceforge.net/) for quality control. Clean reads were obtained using BWA software, and these were aligned with the Setaria italica v2.2 genome sequence (https://phytozome.jgi.doe.gov/pz/portal.html) (Bennetzen et al. 2012) using RapMap.

The RPKM algorithm was used to calculate gene expression, and then conducted differential expression analysis using the DESeq Bioconductor package. Benjamini and Hochberg approach was applied to control the false discovery rate and $p$ value of $<0.05$ was considered for detecting DEGs. GO-TermFinder was used to identify Gene Ontology (GO) terms for annotation of enriched genes with a significant $P$-value of less than 0.05 (Mortazavi et al. 2008).

On the basis of the results obtained, previously reported 16 genes form other crops associated with pollen development were screened out. The relevant sequences were downloaded from the Phytozome database (https://phytozome.jgi.doe.gov/pz/portal.html), from which specific primers were designed. The cDNAs generated following transcriptome analysis were used as templates for qRT-PCR to verify the identified expression profiles. qRT-PCR was performed using an ABI PRISM 7500 Real-Time PCR System (Applied Biosystems, USA) with the cycling parameters: $95^{\circ} \mathrm{C}$ for $30 \mathrm{~s}$, followed by 40 cycles of $95^{\circ} \mathrm{C}$ for $5 \mathrm{~s}, 60^{\circ} \mathrm{C}$ for $34 \mathrm{~s}$, and $72^{\circ} \mathrm{C}$ for $40 \mathrm{~s}$, with a final elongation at $72^{\circ} \mathrm{C}$ for 3 mins.

The actin gene (GenBank: GQ339766.1) was used as an internal control. All reactions were performed with at least three replicates. Results were analyzed using Student's $t$-tests. Statistically significant differences are indicated by asterisk $(P<0.05)$.

\section{Results and Discussion}

Pollens of both untreated and SQ-1-treated plants were stained with $1 \% \mathrm{KI}_{-} \mathrm{I}_{2}$ in order to assess the efficacy of SQ-1. Microscopic observations revealed that the pollen grains of treated plants had stained pale yellow (Fig. 1A), whereas those of control plants had stained dark blue (Fig. 1B). It was found that $96.7 \%$ of the pollen of SQ-1-treated plants were sterile, thereby confirming the efficacy of SQ-1 in inducing male sterility in foxtail millet (Fig. 1C). Significant 
differences were observed between the control and treated plants for most of the agronomic traits following SQ-1 treatment (Fig. 1D: a-e). Agronomic traits namely, plant height, panicle length, panicle width, panicle weight, and grain number per panicle were significantly reduced in treated plants when compared to the control plants, (Fig. 1D: f). Among these differences, panicle weight and grain weight per panicle showed significant marked reduction of 72.5 and $84.8 \%$, respectively, whereas plant height, panicle length, and panicle width decreased 5.3, 6.1 and 14.6\%, respectively.

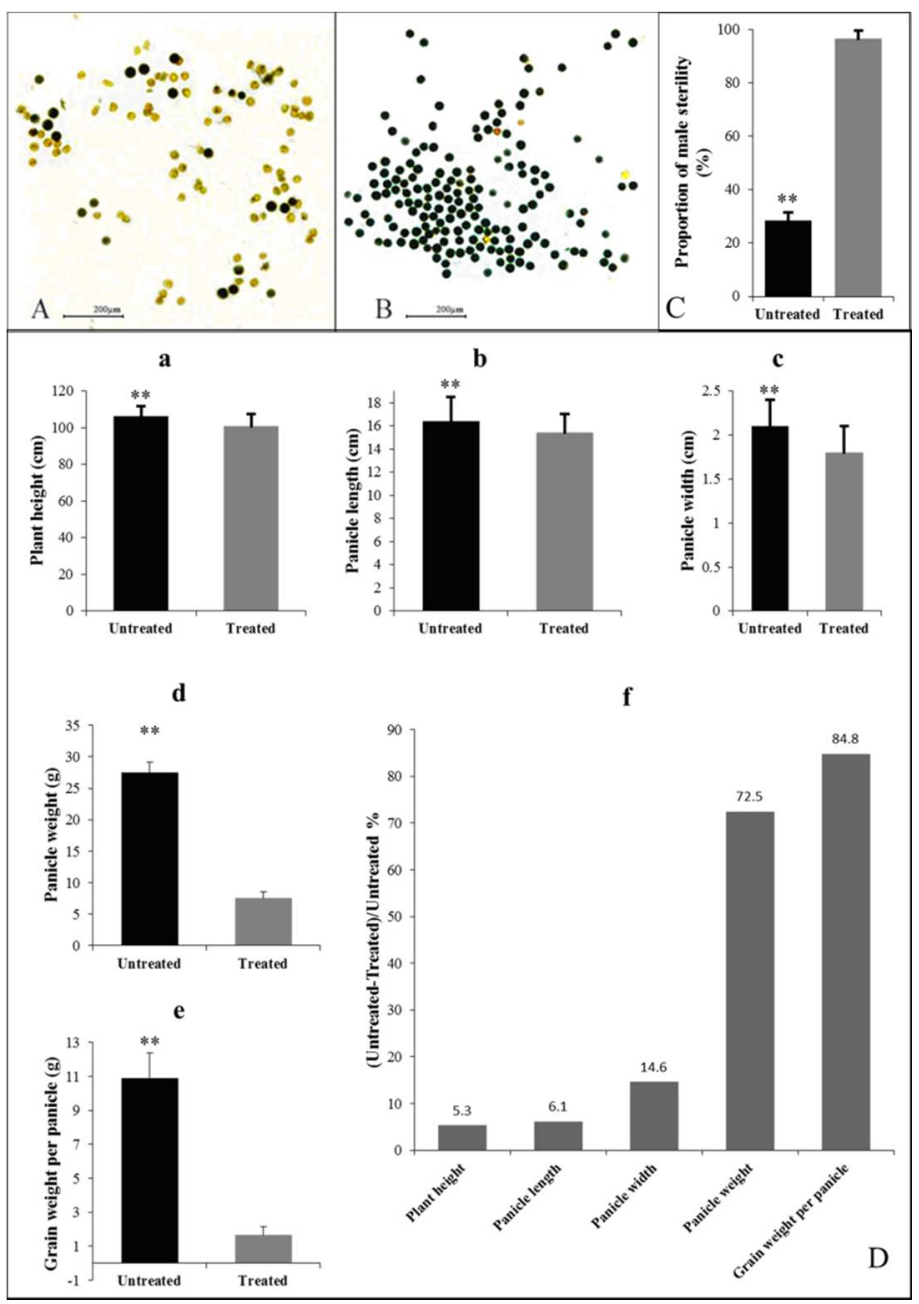

Fig. 1. Mature pollen of control and SQ-1-treated foxtail millet plants. A: Pollen of SQ-1-treated plants stained with 1\% $\mathrm{KI}-\mathrm{I}_{2}$; B: Pollen of untreated plants stained with $1 \% \mathrm{KI}_{2} \mathrm{I}_{2}$. C: Comparison of the proportions of male sterility in control and treated plants; D: Effects of SQ-1 treatment on plant height (a), panicle length (b), panicle width (c), panicle weight (d), grain weight per panicle (e), and the extent of variation of these five agronomic traits (f). ** indicates a significant difference at the 0.01 level, as determined using a $t$-test.

mRNA sequencing libraries were constructed using the immature inflorescences of both SQ-1 treated and control group plants collected at 2nd, 4th and 6th days of treatment. mRNA of the immature inflorescences from SQ-1 treated and control group plants were isolated based on two independent biological replicates. A total of 6659 DEGs were identified from the 2nd day plants, 
among which 3532 and 3127 were up- and down-regulated, respectively. And 1584 and 2654, were detected from the plants of 4th and 6th days of treatment, respectively (Fig. 2A). Thus, the lowest number of DEGs were detected from 4th day plants, among which, the number of downregulated genes were considerably less than that of the up-regulated genes. The number of DEGs slightly increased in 6th day plants; however, at this time point, the number of up-regulated genes were smaller than that of the down-regulated genes. Venn diagram analysis of the DEGs at the three time points resulted that 4928, 227, and 1398 DEGs were pertained to the plants of 2nd, 4th and 6th days of treatment, respectively, while 432 DEGs were common (Fig. 2B). The DEGs shown in the volcano plots presented in Fig. 2C,D,E clearly reveal the changes in expression and significant differences in the fold changes of gene expression among the different samples, thereby indicating the reliability of the data.

A

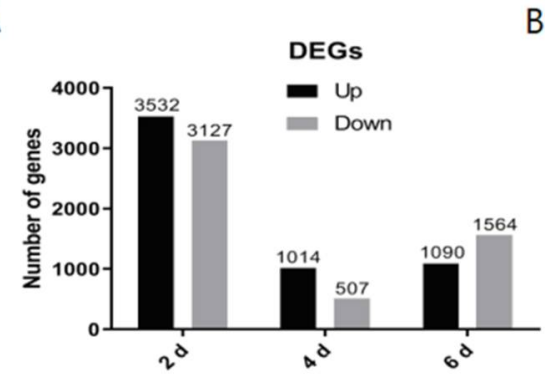

C

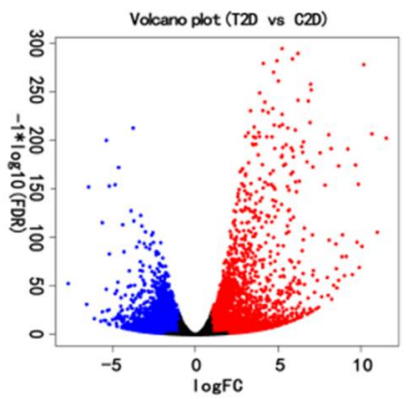

B

D

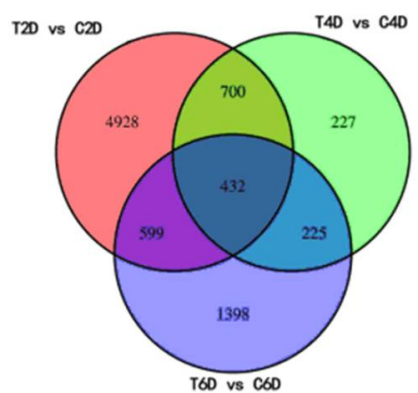

$\mathrm{E}$

Fig. 2. Genes showing differential expression on the $2^{\text {nd }}, 4^{\text {th }}$, and $6^{\text {th }}$ days after SQ-1 treatment. A: Number of differentially expressed genes (DEGs). B: Venn diagram analysis of DEGs at different time points. C-E: Volcano plots of DEGs of the $2^{\text {nd }}, 4^{\text {th }}$, and $6^{\text {th }}$ days after SQ- 1 treatment. DEGs denoted by red and blue points indicate significant differences in up-regulation and down-regulation, respectively. The abscissa indicates the fold changes in gene expression in different samples, whereas the ordinate indicates the statistical significance of the differences in gene expression.

To gain insights into the functions of DEGs, enrichment analysis was performed using the web-based tool agriGO (http://bioinfo.cau.edu.cn/agriGO/index.php). GO-BP functional enrichment analysis revealed that the DEGs that were down-regulated in 2nd day plants were involved in flower development ("sepal formation" and "petal formation"), DNA modification ("DNA methylation" and "histone H3-K9 methylation"), and cell process ("G2/M transition of mitotic cell cycle," "cytokinesis by cell plate formation," and "microtubule-based movement"). Down-regulated DEGs of 6th day plants were mainly involved cell wall organization ("glucuronoxylan metabolic process" and "xylan biosynthetic process"), phenylpropanoid metabolic process ("cinnamic acid biosynthetic process"), and oxidation-reduction. The upregulated DEGs were mainly enriched in "response to stimulus," including "response to chemical 
stimulus," "response to heat," "response to inorganic substance," and "response to reactive oxygen species" (Fig. 3). These results indicated that genes related to flower development were suppressed in response to SQ-1 treatment, whereas certain stress resistance genes became activated. These DEGs thus indicate that male gametocide treatment affects the regulation of metabolic activity and the physiological and biochemical processes associated with stimulus responses.
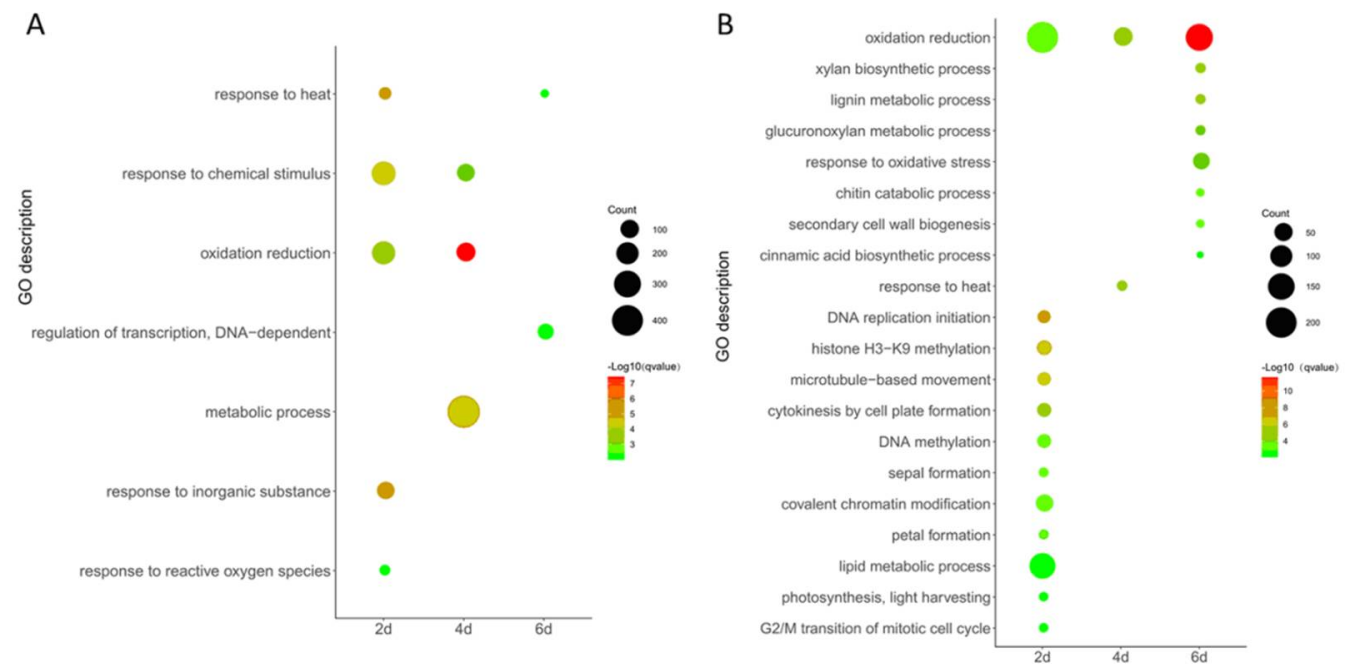

Fig. 3. GO analysis of differentially expressed genes, at 2nd, 4th and 6th days of SQ-1 treatments. A: Up-regulated genes based on GO analysis. B: Down-regulated genes based on GO analysis.

In the present study, 16 DEGs referred to other crops to verify their homogous genes' expression between the SQ-1-treated and control plants were used. These genes included DFR2A (Sachiko et al. 2013), ABCB1 (Cecchetti et al. 2015), NEFl (Ariizumi et al. 2004), Msl (Yang et al. 2007) which are involved in 'anther' development along with other genes such as GH3 (Feng et al. 2015), FD (Kaur et al. 2021), MKK4/5P (Zhang et al. 2016). Gene expression was measured by qRT-PCR using gene-specific primers. Among them, eight up-regulated (Fig. 4A) and eight down-regulated (Fig. 4B) genes were validated.

The expression of those genes, detected by qRT-PCR, showed significant up- or downregulation, which were consistent with the aforementioned results obtained from transcriptome analysis. Among these genes, the expression of sulfite reductase (Ferredoxin, FD) (Seita.3G187400) increased after treatment with chemical hybridization agents (Fig. 4A). Furthermore, transcriptome and qRT-PCR analyses validated the SQ-1-induced enhanced expression of SiGh3 (Seita.2G375300.1), an auxin early response gene (Fig. 4A). The expression of SiAUT3 (Seita.4G026700.1) was significantly reduced in response to SQ-1 treatment in foxtail millet (Fig. 4B). Male sterility caused by $M S$ gene mutations was reported in many crops. $M s l$ is required for pollen wall biosynthesis in Arabidopsis (Yang et al. 2007), the expression of homologous SiMs1 (Seita.2G224700.1) in foxtail millet was found to reduce after SQ-1 treatment, which might also lead to male sterility. Further analysis and verification are necessary for other genes that are differentially expressed after SQ-1 treatment to determine those genes that are responsible for male sterility in foxtail millet. 
A
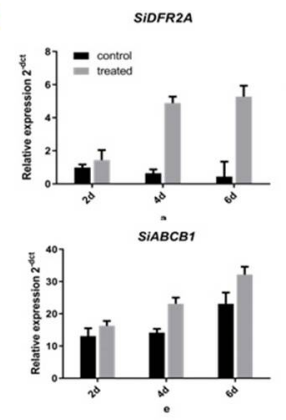

B

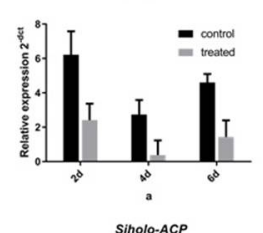

Siholo-ACP

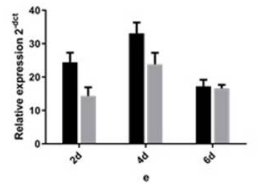

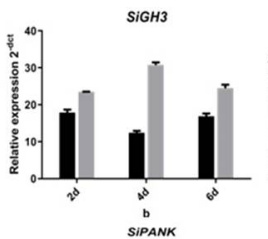
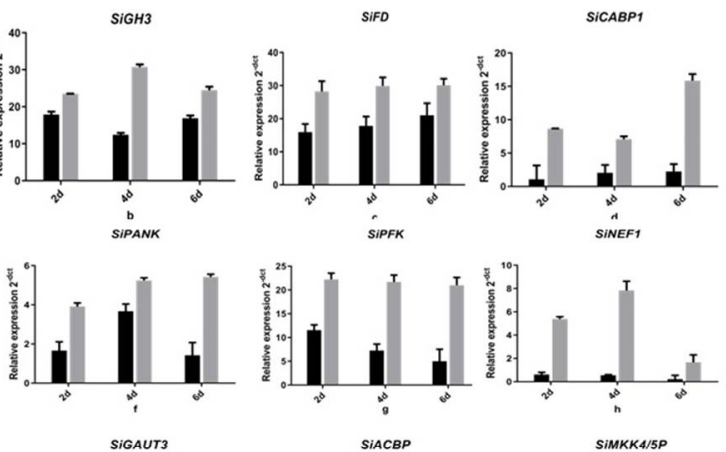

SIGAUTS
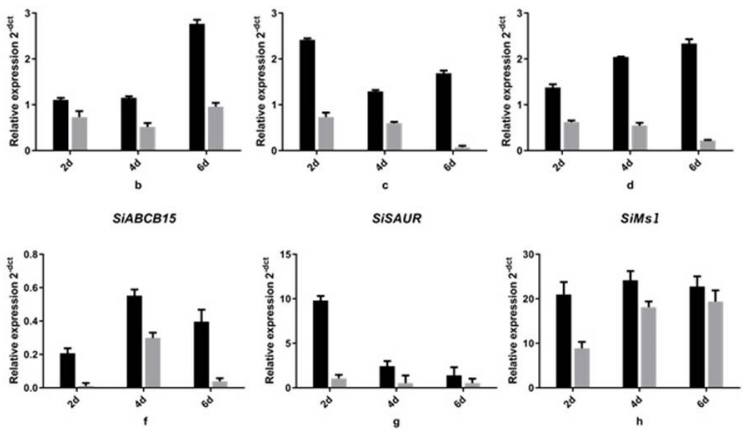

Fig. 4. qRT-PCR expression analysis of differentially expressed (DEGs) genes between control and treatment samples. A: Up-regulated DEGs. B: Down-regulated DEGs.
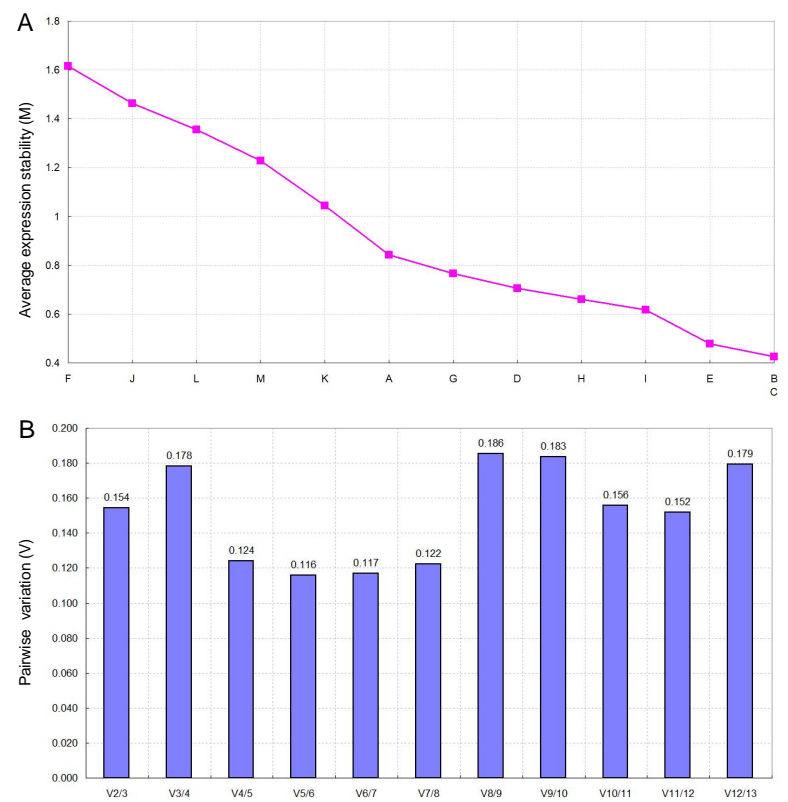

Fig. 5. Reference gene validation under SQ-1 treatment. A. Analysis of the expression stability of candidate reference genes by geNorm. The reference genes were shown instead of A-M and the genes ID were listed as follow: A: Si000245, B: SiO02651, C: SiO02929, D: Si014034, E: Si017354, F: Si018607, G: SiO18608, H: SiO21145, I: SiO21373, J: SiO22372, K: Si030042, L: Si034613, M: Si035654. B. Determined the optimal number of candidate reference genes by geNorm. 
Many studies have reported that reference genes can differ under various conditions. In the present study, 13 reference genes (SiO00245, SiO02651, SiO02929, SiO14034, SiO17354, SiO18607, Si018608, Si021145, Si021373, Si022372, Si030042, Si034613, Si035654) were chosen from previous researches to confirm the reliability of SQ-1 treatment. The Cq values of the reference genes were variable from real-time PCR. The $\mathrm{M}$ values were calculated by geNorm to determine the stability of the reference genes. Besides, the lower $\mathrm{M}$ values the expression of the gene was more stable under SQ-1 treatment. However, reference genes SiO02651 and SiO02929 were found to be the most proper option for SQ-1 treatment (Fig. 5A). The pairwise change (V) was also calculated and the values of $V \mathrm{n} / \mathrm{Vn}+1$ determined the optimal amount by geNorm. Results showed that $V 2 / V 3=0.154>0.15$ (Fig. 5B), therefore, three genes were sufficient for use in normalizing gene expression in SQ-1 treatment.

The present study is the first to compare the immature inflorescence transcriptomes of foxtail millet in which male sterility has been induced by SQ-1, and a great number of important genes on SQ-1-induced male sterility were identified. In future this finding would contribute to the examination of the genes or pathways related to male fertility in foxtail millet.

\section{Acknowledgments}

This research was supported by the Science and Technology Research Projects of Colleges and Universities in Hebei Province (QN2018330, QN2020505), the Natural Science Foundation of Hebei Province (C2016101019). The authors would like to thank Wei Li at the Institute of Millet Crops, Hebei Academy of Agriculture and Forestry Sciences for providing the foxtail millet variety Yugu 1 and the male gametocide SQ-1.

\section{References}

Ariizumi T, Hatakeyama K, Hinata K, Inatsugi R, Nishida I, Sato S, Kato T, Tabata S and Toriyama K 2004. Disruption of the novel plant protein NEF1 affects lipid accumulation in the plastids of the tapetum and exine formation of pollen, resulting in male sterility in Arabidopsis thaliana. Plant. J. 39: 170-181.

Bennetzen JL, Schmutz J, Wang H, Percifield R, Hawkins J, Pontaroli AC, Estep M, Feng L, Vaughn JN, Grimwood J, Jenkins J, Barry K, Lindquist E, Hellsten U, Deshpande S, Wang X, Wu X, Mitros T, Triplett J, Yang X, Ye CY, Mauro-Herrera M, Wang L, Li P, Sharma M, Sharma R, Ronald PC, Panaud O, Kellogg EA, Brutnell TP, Doust AN, Tuskan GA, Rokhsar D and Devos KM 2012. Reference genome sequence of the model plant Setaria. Nat. Biotechnol. 30: 555-561.

Cecchetti V, Brunetti P, Napoli N, Fattorini L, Altamura MM, Costantino P and Cardarelli M 2015. ABCB1 and ABCB19 auxin transporters have synergistic effects on early and late Arabidopsis anther development. J. Integr. Plant. Biol. 57:1089-1098.

Feng S, Yue R, Tao S, Yang Y, Zhang L, Xu M, Wang H and Shen C 2015.Genome-wide identification, expression analysis of auxin-responsive GH3 family genes in maize (Zea mays L.) under abiotic stresses. J. Integr. Plant Biol. 57:783-795.

Kaur A, Nijhawan A, Yadav M, Khurana JP 2021. OsbZIP62/OsFD7, a functional ortholog of FLOWERING LOCUS D (FD), regulates floral transition and panicle development in rice. J. Exp. Bot., erab396, https://doi.org/10.1093/jxb/erab396.

Mortazavi A, Williams BA, McCue K, Schaeffer L and Wold B 2008. Mapping and quantifying mammalian transcriptomes by RNA-Seq. Nat. Methods 5: 621-628.

Sachiko S, Yasuko K, Reina K, Hiroyasu K, Minoru N and Takeshi N 2013. Identification of a single nucleotide deletion causing frameshift mutation in $O s D F R 2 A$ in a genic male sterile mutant of rice and its possible application to F1 hybrid breeding. Mol. Breeding 31: 805-814.

Song Y, Wang L, Zhang G, Ying S and Zhao Z 2011. Male sterility induced by chemical hybridizing agent SQ-1 in Setaria italica Beauv. Acta Agron. Sin.(China) 37: 1695-1700. 
Wang CF, Zhang WD, Li TC, Xiong X, Gu SQ, Li W and Shi SK 2019. Effect of physiological and biochemical indexes on young panicles under chemical hybridization agent SQ-1 in foxtail millet. J. Hubei Univ., Nat. Sci. Ed. 39: 404-411.

Wang RQ, Gao JH, Mao LP, Du RH, Diao XM and Sun JS 2002. Chromosome location of the male-sterility and yellow seedling gene in line 1066A of foxtail millet. Acta Bot. Sin. 44: 1209-1212.

Wang SP, Zhang GS, Song QL, Zhang YX, Li Y, Guo JL, Chen Z, Niu N, Ma SC and Wang JW 2016. Programmed cell death, antioxidant response and oxidative stress in wheat flag leaves induced by chemical hybridization agent SQ-1. J. Integr. Agric. 15: 76-86.

Wang ZH, Liu HW, Zhang GS, Wang JW and Ma SC 2003. Effects on male sterility induced by three kinds of chemical hybridization agent in wheat. J. Northwest Sci. Tech. Univer. Agric. Forest. 31: 43-46.

Yang CY, Vizcay-Barrena G, Conner K and Wilson ZA 2007. MALE STERILITY1 is required for tapetal development and pollen wall biosynthesis. Plant Cell 19: 3530-3548.

Zhang YM, Liu ZH, Yang RJ, Li GL, Guo XL, Zhang HN, Zhang HW, Di R, Zhao QS and Zhang MC 2016. Improvement of soybean transformation via Agrobacterium tumefaciens methods involving $\alpha$ aminooxyacetic acid and sonication treatments enlightened by gene expression profile analysis. Plant Cell Rep. 35:1259-71.

Zhu Q, Song Y, Zhang G, Ju L, Zhang J, Yu Y, Niu N, Wang J and Ma S 2015. De novo assembly and transcriptome analysis of wheat with male sterility induced by the chemical hybridizing agent SQ-1. Plos one 10: e0123556.

(Manuscript received on 6 July, 2021; revised on 13 September, 2021) 